\section{Study on antibacterial and flavonoid content of ethanolic extract of Punica granatum (pomegranate) peel}

\author{
Renu Narendra Jaisinghani, ${ }^{1}$ \\ Shweta Makhwana, ${ }^{2}$ Ankit Kanojia ${ }^{2}$ \\ ${ }^{1}$ Department of Microbiology, Smt. \\ Chandibai Himmatmal Mansukhani \\ (CHM) College, Mumbai; ${ }^{2}$ Department \\ of Microbiology, Jai Hind College, \\ Mumbai, India
}

\begin{abstract}
Pomegranate is known for it's many health benefits. These benefits are due to the biological active compounds which are present in the pulp as well as in the peel of the pomegranate. The active compounds from the peel were obtained using cold percolation method using ethanol as solvent. Antibacterial activity of pomegranate peel extract (PPE) was studied on E.coli, E. coli NCIM 2065, Salmonella typhi, Salmonella paratyphi B, Pseudomonas aeruginosa, Proteus mirabilis, $S$. aureus, $S$. aureus NCIM2079, Shigella flexneri, Lactobacillus casei var shirota by agar well diffusion method. All organisms were sensitive to the extract with inhibitory concentration ranging from $25 \mathrm{mg} / \mathrm{mL}-100 \mathrm{mg} / \mathrm{mL}$ except Lactobacillus casei var shirota which was resistant to even $100 \mathrm{mg} / \mathrm{mL}$ concentration of PPE. Flavonoid content of the extract was found to be $10 \mathrm{mg}$ quercetin equivalent/g of extract.
\end{abstract}

\section{Introduction}

Pomegranate (Punica granatum) belongs to the Punicaceae family and is a nutrient dense food source rich in phytochemical compounds. Pomegranates are popularly consumed as fresh fruit, beverages, food products (jams and jellies), and extracts wherein they are used as botanical ingredients in herbal medicines and dietary supplements. Several studies reported that phytochemicals have been identified from various parts of the pomegranate tree and from pomegranate fruit: peel, juice, and seeds. ${ }^{1}$

Pomegranate polyphenols include flavonoids (flavonols, flavanols, anthocyanins.), condensed tannins (proanthocyanidins), and hydrolyzable tannins (ellagitannins and gallotannins). Other phytochemicals identified from the pomegranate are organic and phenolic acids, sterols and triterpenoids, fatty acids, triglycerides, and alkaloids. ${ }^{1}$ Other major components of pomegranate juice are ellagic, caffeic, and punicic acids. These phenolic compounds belong to different representative chemical classes with known bioactivities.

Apart from flavonoids and tannins pomegranate also contains anthocyanins. Anthocyanins possess known pharmacological properties and are used by humans for therapeutic purposes. ${ }^{2}$ Anthocyanins are the water-soluble pigments responsible for the bright red color of pomegranate. Several anthocyanin compounds identified in pomegranate, include pelargonidin-3-glucoside, cyanidin-3-glucoside, delphinidin3-glucoside, pelargonidin 3,5-diglucoside, cyanidin 3,5-diglucoside and delphinidin 3,5-diglucoside. ${ }^{3}$ Anthocyanins can be insect attractants in flowers but can also be insecticidal and antimicrobial at the same time. Tannins as well as anthocyanins have significant antiproliferative and proapoptotic effects in several different types of cancer cells in vitro, including colon cancer, prostate cancer, and head and neck cancer. ${ }^{4}$

Peak levels of these bioactives are found in fruit peel then in the pulp. The peels of fruits are discarded thereby increasing solid waste production, even though they are actually rich in polyphenols. Thus, this waste which is mine of nutraceuticals can be harnessed for its fullest potential. The peels of pomegranate contain 249.4 $\mathrm{mg} / \mathrm{g}$ of phenolic compounds as compared to only $24.4 \mathrm{mg} / \mathrm{g}$ phenolic compounds found in the pulp of pomegranate. ${ }^{5}$

The probiotics are live microorganisms which exert a beneficial effect on the health of the host when they are administered in adequate quantities (FAO/WHO, 2002) ${ }^{6}$ The consumption of living or lyophilized cultures of probiotic bacteria improves the immune system action, prevents cancer, atherosclerosis and coronary diseases. The beneficial effect of probiotics on diarrhoea, gastroenteritis, irritable bowel syndrome, inflammatory bowel disease, lactose digestion, infant allergies, hyperlipidaemia, hepatic disease, Helicobacter pylori infections is well proved. 7,8

Functional foods are those foods that provide benefits beyond basic nutrition when consumed as part of the regular diet and help in prevention and treatment of illness and disease. ${ }^{9}$ When the probiotic bacteria and phytochemicals are combined a product can be developed having double benefits packed in a single product for health and wellbeing.

Thus, the present study evaluates effect of ethanolic pomegranate peel extract on Gram positive and Gram negative pathogenic organisms. And it's effect on probiotic bacteria L. casei var shirota.
Correspondence: Renu Narendra Jaisinghani, Smt. Chandibai Himathmal Mansukhani (CHM) College, Ulhasnagar, Dist: Thane. Mumbai 421003. India.

Tel.: +91.9320512121.

E-mail : renu.nj24@gmail.com

Key words: Antibacterial; Lactobacillus casei var shirota; Flavonoids.

Acknowledgements: this work was carried out during my tenure at Jai Hind College, Mumbai. I am thankful to the entire Microbiology Department with special thanks to Dr. M.S. Ghayal.

Received for publication: 4 November 2017 Revision received: 3 February 2018.

Accepted for publication: 16 February 2018.

This work is licensed under a Creative Commons Attribution NonCommercial 4.0 License (CC BY-NC 4.0).

(C) Copyright R.N. Jaisinghani et al., 2018

Licensee PAGEPress, Italy

Microbiology Research 2018; 9:7480

doi:10.4081/mr.2018.7480

\section{Materials and Methods}

\section{Materials}

Pomegranates were procured from the local market of Mumbai, Maharashtra, India. All the chemicals (viz, Quercetin, Aluminium Chloride, Dimethyl sulphoxide (DMSO) ethanol and methanol) were of analytical grade and were procured from Sigma Aldrich, Mumbai, India. Sterile Muller and Hinton Broth, Sterile MRS broth, Sterile Nutrient Agar were procured from Hi Media, India.

\section{Test organisms}

The cultures from Culture Collection of Department of Microbiology, Faculty of Science, Jai Hind College, Mumbai were used in the study. The organisms used comprise of six Gram-negative organisms (Escherichia coli, Salmonella typhi, Salmonella paratyphi B, Shigella flexneri, Proteus mirabilis, Pseudomonas aeruginosa) and two Gram positive organisms (Staphylococcus aureus, Lactobacillus casei var shirota).

\section{Control organisms}

Control strains of Staphylococcus aureus NCIM 2079 and Escherichia coli NCIM2065 were used and tested along with the organisms. 


\section{Preparation of extract}

The arils of pomegranate were removed and the peel along with albedo was used for preparation of peel extract. The peels were carefully washed under running tap water followed by sterile distilled water. These were air dried at $\left(45^{\circ} \mathrm{C}\right)$ for two days, pulverized to a fine powder using a sterilized mixer grinder and stored in air-tight bottles. For the purpose of extraction, a $10 \mathrm{~g}$ amount of the pulverized peel was soaked in $90 \mathrm{~mL}$ of ethanol $(96 \%)$ for $24 \mathrm{~h}$. After $24 \mathrm{~h}$ the extract was filtered through Whatman filter paper No.1 for removal of peel particles and concentrated under vacuum below $40^{\circ} \mathrm{C}$ using Heidolph, VE-11 rotaevaporator. The dried extract thus obtained was exposed to $\mathrm{UV}$ rays for $2 \mathrm{~h}$ and checked for sterility on nutrient agar plates and stored in labelled sterile bottles at $4^{\circ} \mathrm{C}$ until further use.

\section{Standardisation of inoculum}

Bacterial strains were maintained on Sterile Nutrient agar slopes. Bacterial strains were first grown on Mueller Hinton Medium for 18 to $24 \mathrm{~h}$ at $37^{\circ} \mathrm{C}$. The inoculums of the indicated bacterial strains were transferred into physiological suspension medium and adjusted to 0.5 Mac Farland turbidity standard $\left(10^{8} \mathrm{cfu} / \mathrm{mL}\right)$.

The strain of Lactobacillus casei var shirota was isolated on Sterile MRS agar from commercially available probiotic drink Yakult. Incubated at $30^{\circ} \mathrm{C}$ under anaerobic condition. The purified culture was preserved on sterile MRS agar slants. The inocula of L.casei var shirota was prepared in the similar way as described above.

\section{Antibacterial assay}

Antibacterial assay was carried out by using agar well diffusion method. ${ }^{10}$ Sterile molten Mueller and Hinton at around $40^{\circ} \mathrm{C}$ was taken and seeded with different micro- bial cultures and plates were prepared. After solidification $6 \mathrm{~mm}$ wells were punched. In these wells peel extract dissolved in $10 \%$ DMSO at different dilutions were added (100 mg/mL, $50 \mathrm{mg} / \mathrm{mL}, 25 \mathrm{mg} / \mathrm{mL}$ ). The plates were incubated overnight at $37^{\circ} \mathrm{C}$. After incubation, the zones of inhibition were measured and recorded. Controls were also simultaneously performed. All tests were carried out in triplicates.

\section{Determination of total flavonoid content}

Total flavonoid content was estimated by Aluminium chloride colorimetric method. ${ }^{10,11}$ The principle involved in Aluminium chloride $\left(\mathrm{AlCl}_{3}\right)$ colorimetric method is that $\mathrm{AlCl}_{3}$ forms acid stable complexes with the $\mathrm{C}-4$ keto groups and either the C-3 or C-5 hydroxyl group of flavones and flavonols. In addition, it also forms acid labile complexes with the orthodihydroxyl groups in the A- or B-ring of flavonoids. Briefly, $0.5 \mathrm{~mL}$ solution of diluted pomegranate peel extract in methanol was separately mixed with $1.5 \mathrm{~mL}$ of methanol, $0.1 \mathrm{~mL}$ of $10 \%$ Aluminium Chloride, 0.1 $\mathrm{mL}$ of $1 \mathrm{M}$ potassium acetate, and $2.8 \mathrm{~mL}$ of distilled water, and left at room temperature for 30 minutes. The absorbance of the reaction mixture was measured at $415 \mathrm{~nm}$ with a double beam Systronic UV/Visible spectrophotometer. Total flavonoid content was calculated as quercetin from a calibration curve. The calibration curve was prepared by preparing quercetin solutions at concentrations 12.5 to $100 \mathrm{mcg} / \mathrm{mL}$ in methanol. ${ }^{12}$

\section{Statistical analysis}

The data obtained were analyzed using SPSS (Statistical Package for Social Sciences) version 11.5 (SPSS Inc., Chicago, Illinois, USA). Descriptive statistics (Mean value and $\mathrm{SD}$ ) along with comparison in mean zone of inhibition between the extract at different concentrations on different organisms were performed using One Way Analysis of Variance (ANOVA). Confidence level and level of significance were set at $95 \%$ and $5 \%$ respectively.

\section{Results and Discussion}

\section{Antibacterial activity of extract}

In the present study, a good antibacterial activity of ethanolic extract of pomegranate peel was observed. Zone of inhibition of the extract (in $\mathrm{mm}$ ) significantly increased $(p \leq 0.05)$ as the concentration of the extract increased. Of the ten organisms used in the study nine were inhibited by the PPE; at concentration range $100-25 \mathrm{mg} / \mathrm{mL}$. Highest Zone of inhibition were seen in given order Shigella flexineri $>$ Salmonella typhi $>$ Staphylococcus aureus $>$ Salmonella typhi paraB $\geq$ Pseudomonas aeruginosa $>$ Proteus mirabilis $\geq$ Staphylococcus aureus NCIM2079 > Escherichia coli >Escherichia coli NCIM2065. While Lactobacillus casei var shirota was resistant to the extract. The Mean zone of inhibition (mm) of ethanol extract of pomegranate (Punica granatum) peel are given in (Table 1). The results are in agreement with earlier investigations reported by other authors to inhibit different microorganisms. Effect of PPE on Bacillus subtilis, Staphylococcus aureus, Escherichia coli, and Salmonella typhimurium has been reported. The MIC for ethanol extract of peels was $242-500 \mathrm{mg} / \mathrm{mL}$ against all test bacteria. ${ }^{13}$ Al-Zoreky found that methanolic extract of pomegranate fruit peels is a potent inhibitor for Staphylococcus aureus, Listeria monocytogenes, Escherichia coli and Yersinia enterocolitica. ${ }^{14}$ In another study by (Sadeghian et al,2011) aqueous and methanolic extracts of pomegranate

Table 1. Mean zone of inhibition (mm) of ethanol extract of Pomegranate (Punica granatum) peel.

\begin{tabular}{|c|c|c|c|c|c|}
\hline \multirow[t]{2}{*}{ Sr. No. } & \multirow[t]{2}{*}{ Organism } & \multicolumn{3}{|c|}{ Concentration (Mean Zone of Inhibition \pm SD) } & \multirow[t]{2}{*}{ p-value } \\
\hline & & $100 \mathrm{mg} / \mathrm{mL}$ & $50 \mathrm{mg} / \mathrm{mL}$ & $25 \mathrm{mg} / \mathrm{mL}$ & \\
\hline 1 & Salmonell typhi & $28.33 \pm 1.52$ & $25.33 \pm 1.52$ & $23.33 \pm 1.15$ & 0.0138 \\
\hline 2 & Salmonella paratyphi $B$ & $26 \pm 1$ & $24.33 \pm 1.15$ & $22 \pm 1$ & 0.0100 \\
\hline 3 & Escherichia coli & $23.33 \pm 1.52$ & $21 \pm 1$ & $19.33 \pm 1.52$ & 0.0323 \\
\hline 4 & Shigella flexineri & $30.33 \pm 1.52$ & $23 \pm 1$ & $22.66 \pm 1.15$ & 0.0004 \\
\hline 5 & Proteus mirabilis & $24 \pm 1$ & $22 \pm 1$ & $21.66 \pm 0.57$ & 0.0353 \\
\hline 6 & Pseudomonas aeruginosa & $26 \pm 1$ & $23 \pm 1$ & $22 \pm 1$ & 0.0065 \\
\hline 7 & Staphylococcus aureus & $26.66 \pm 1$ & $23.66 \pm 0.577$ & $20.66 \pm 1.52$ & 0.0038 \\
\hline 8 & Escherichia coli NCIM2065 & $19.66 \pm 0.57$ & $19.33 \pm 0.57$ & $17.66 \pm 0.57$ & 0.0110 \\
\hline 9 & Staphylococcus aureus NCIM2079 & $24 \pm 1$ & $20 \pm 1$ & $23.33 \pm 0.57$ & 0.0030 \\
\hline 10 & Lactobacillus casei var shirota & $\mathrm{R}$ & $\mathrm{R}$ & $\mathrm{R}$ & - \\
\hline
\end{tabular}


fruit skin showed antimicrobial activity against Staphylococcus aureus, Pseudomonas aeruginosa and Candida albicans. ${ }^{15}$ In the study carried by Sangeetha et al. antimicrobial activity of Ganesh and Kabul varieties of pomegranate peel extract were evaluated. In the study, they found that Kabul variety the ethyl acetate extract was effective against Bacillus, E.coli, Pseudomonas, Staphylococcus and Candida. The Aqueous extract was effective against Aeromonas, Bacillus, E.coli, Pseudomonas, Staphylococcus, Vibrio and Candida. The methanolic extract of Ganesh variety of Punica granatum showed increased antimicrobial activity against each of the tested strains, and the values ranged from 31.75 to $1000 \mathrm{mcg} / \mathrm{mL}$ and in case of Kabul variety the ethyl acetate extract showed activity varying from 62.5 to $500 \mathrm{mcg} / \mathrm{mL}$ for selected strains. ${ }^{16}$ In the work carried by Malviya et al. showed antibacterial activity of ethanol peel extract of pomegranate of Ganesh variety on Staphylococcus aureus, Enterobacter aerogenes, Salmonella typhi and Klebsiella pneumoniae but concentration at which they are inhibited is not mentioned. ${ }^{17}$

Pomegranate peel polyphenols, especially tannins are the major components in the pomegranate peel extract that have been implicated in antimicrobial potential i.e. antiviral, antifungal and antibacterial activities. ${ }^{3}$ In the study by Vasconcelos antibacterial activity of methanolic peel extracts of pomegranate cultivars against both Gram negative and positive bacteria strains have been reported. The MIC values were found to be ranging from 0.25 to $4.0 \mathrm{mg} / \mathrm{mL}$. The author has reported a two-fold MIC value against Staphylococcus aureus than against Escherichia coli. ${ }^{18}$ It has been suggested that the antimicrobial activity of tannins may be due to the ability of tannin compounds to precipitate proteins, therefore causing leakage of cell membrane of the microorganism, and aiding cell lysis which ultimately leads to cell death. ${ }^{19}$ As reported by Olaniyi et al. Methanolic pomegranate peel extract showed strong broad-spectrum activity against Gram-positive and Gramnegative bacteria, with the minimum inhibitory concentrations (MIC) ranging from 0.2 to $0.78 \mathrm{mg} / \mathrm{mL} .{ }^{20}$

As documented in the earlier studies the effect of methanol peel extract is done by major researchers. With little work on ethanol extract, here we are reporting that even ethanol pomegranate peel extract is able to inhibit both Gram positive and Gram negative bacteria.

\section{Effect on Lactobacillus casei var shi- rota}

Lactobacillus casei var shirota showed resistance to the PPE at concentration of $100 \mathrm{mg} / \mathrm{mL}$. Bialonska et al. has reported; effect of pomegranate tannin constituents on the growth of various species of human gut bacteria in vitro. The pomegranate by products and punicalagins inhibited the growth of pathogenic Clostridia and Staphyloccocus aureus. Probiotic Lactobacilli and Bifidobacteria were generally not affected by ellagitannins, while a relatively small growth inhibition by ellagic acid likely resulted from decreasing media quality due to the formation of tannin-protein complexes. ${ }^{21}$ In an investigation found that intake of flavonol-rich foods can modify the composition of the gut microbiota, exerting prebiotic-like effects. ${ }^{22}$ Unabsorbed dietary phenolics and their metabolites have been shown to exert antimicrobial or bacteriostatic activities. These metabolites selectively inhibit pathogen growth and stimulate the growth of commensal bacteria, including also some recognized probiotics thus influencing the microbiota composition. ${ }^{23}$

\section{Flavonoid content}

The flavonoid content of the pomegranate peel extract was found to be $10 \mathrm{mg}$ quercetin equivalent/g of extract. Flavonoids are known to show antioxidant activity having considerable effects on human nutrition and health. The mechanism of flavonoid action is based on scavenging or chelating process. ${ }^{24}$

\section{Conclusions}

From the study undertaken it can be concluded that ethanolic extract of pomegranate peel has antibacterial properties and good flavonoid content. These properties can be used to formulate new products to be used in food industry as natural antioxidant, replacing synthetic antioxidants, and also as natural food preservatives. Also, resistance of Lactobacillus casei var shirota to PPE makes it a good candidate to be used as an ingredient in preparation of functional foods.

\section{References}

1. Adams LS, Seeram NP, Aggarwal BB, Takada Y, Sand D, Heber D. Pomegranate juice, total pomegranate ellagitannins, and punicalagin suppress inflammatory cell signaling in colon cancer cells. Journal of Agricultural and Food Chemistry. 2006 Feb 8;54(3):9805.

2. Porter ML, Krueger CG, Wiebe DA, Cunningham DG, Reed JD. Cranberry proanthocyanidins associate with low-density lipoprotein and inhibit in vitro $\mathrm{Cu} 2+$-induced oxidation. Journal of the Science of Food and Agriculture. 2001 Nov 1;81(14):1306-13.

3. Miguel MG, Neves MA, Antunes MD. Pomegranate (Punica granatum L.): A medicinal plant with myriad biological properties-A short review. Journal of Medicinal Plants Research. 2010 Dec 29;4(25):2836-47.

4. Elfalleh W, Tlili N, Nasri N, Yahia Y, Hannachi H, Chaira N, Ying $M$, Ferchichi A. Antioxidant capacities of phenolic compounds and tocopherols from Tunisian pomegranate (Punica granatum) fruits. Journal of food science. 2011 Jun 1;76(5):C707-13.

5. Rudra SG, Nishad J, Jakhar N, Kaur C. Food industry waste: mine of nutraceuticals. Int. J. Sci. Environ. Technol. 2015;4(1):205-29.

6. Joint FA.WHO working group report on drafting guidelines for the evaluation of probiotics in food. London, Ontario, Canada.2002 Apr30;30.

7. Brown AC, Valiere A. Probiotics and medical nutrition therapy. Nutrition in clinical care: an official publication of Tufts University. 2004;7(2):56.

8. Marteau PR, de Vrese M, Cellier CJ, Schrezenmeir J. Protection from gastrointestinal diseases with the use of probiotics. The American journal of clinical nutrition. 2001 Feb 1;73(2): 430s-6s.

9. Hasler CM. Functional foods: their role in disease prevention and health promotion. Food technology campaign then Chicago.1998 Nov 1;52:63-147.

10. Collins, C.M, P. Lyne and J. Grange, 1989.Antimicrobial sensitivity and assay tests. Collins and Lyne Microbiological Methods. Butterworths, London, UK.

11. Nabavi SM, Ebrahimzadeh MA, Nabavi SF, Hamidinia A, Bekhradnia AR. Determination of antioxidant activity, phenol and flavonoids content of Parrotia persica Mey. Pharmacology online. 2008;2:560-7.

12. Bag GC, Devi PG. Assessment of total flavonoid content and antioxidant activity of methanolic rhizome extract of three Hedychium species of Manipur 
valley. International Journal of Pharmaceutical Sciences Review and Research. 2015;30(1):154-9.

13. Nuamsetti T, Dechayuenyong P, Tantipaibulvut S. Antibacterial activity of pomegranate fruit peels and arils. Science Asia. 2012 Sep 1;38(3):319-22.

14. Al-Zoreky NS. Antimicrobial activity of pomegranate (Punica granatum L.) fruit peels. International journal of food microbiology. 2009 Sep 15;134(3):2448.

15. Sadeghian A, Ghorbani A, MohamadiNejad A, Rakhshandeh H. Antimicrobial activity of aqueous and methanolic extracts of pomegranate fruit skin. Avicenna J Phytomed. 2011 Sep 1;1:67-73.

16. Sangeetha J, Vijayalakshmi K. Antimicrobial activity of rind extracts of Punica granatum linn. Bioscan. 2011 Feb 21;6(1):119-24.

17. Malviya S, Jha A, Hettiarachchy N.
Antioxidant and antibacterial potential of pomegranate peel extracts. Journal of food science and technology. $2014 \mathrm{Dec}$ 1;51(12):4132-7.

18. Vasconcelos NS: Antimicrobial activity of pomegranate (Punica granatumL.) fruit peels. Int J Food Microbiol 2009, 134:244-248.

19. Johanningsmeier SD, Harris GK. Pomegranate as a functional food and nutraceutical source. Annual review of food science and technology. $2011 \mathrm{Apr}$ 10;2:181-201.

20. Fawole OA, Makunga NP, Opara UL. Antibacterial, antioxidant and tyrosinase-inhibition activities of pomegranate fruit peel methanolic extract. BMC complementary and alternative medicine. 2012 Oct 30;12(1):200.

21. Bialonska D, Kasimsetty SG, Schrader KK, Ferreira D. The effect of pomegranate (Punica granatum L.) byproducts and ellagitannins on the growth of human gut bacteria. Journal of agricultural and food chemistry. 2009 Aug 25;57(18):8344-9.

22. Tzonuis $\mathrm{X}$, Vulevic J, Kuhnle GG, George T, Leonczak J, Gibson GR, et al. Flavanol monomer-induced changes to the human faecal microflora. Br J Nutr 2008;99:782-92.

23. Laparra JM, Sanz Y. Interactions of gut microbiota with functional food components and nutraceuticals. Pharmacological Research. 2010 Mar 31;61(3):219-25.

24. Ruiz PA, Haller D. Functional diversity of flavonoids in the inhibition of the proinflammatory NF-kappaB, IRF, and Akt signaling pathways in murine intestinal epithelial cells. J Nutr 2006;136:664-71. 